\title{
Results of primary versus recurrent surgery to treat stress urinary incontinence in women
}

\author{
Maarten J. van der Doelen • Mariëlla I. J. Withagen • \\ Mark E. Vierhout • John P. F. A. Heesakkers
}

Received: 11 September 2014 / Accepted: 6 January 2015 / Published online: 10 March 2015

(C) The Author(s) 2015. This article is published with open access at Springerlink.com

\begin{abstract}
Introduction and hypothesis We compared cure rates and complication rates in patients who had undergone primary or recurrent (secondary) surgery for stress urinary incontinence (SUI).

Methods A retrospective cohort study that included patients who underwent surgery to treat SUI in a tertiary referral center was carried out. All patients had, predominantly, SUI. Exclusion criteria were patients with a neurogenic bladder or a neobladder and patients without postoperative follow-up (FU). The primary objective was to assess the success rate, defined as cured SUI or improved SUI at six weeks and at the latest available moment of FU. The secondary objective was to assess complications.

Results A total of 541 women with SUI underwent surgery for SUI between 2002 and 2010. After exclusion of 102 patients a total of 242 patients with primary SUI and 197 patients with recurrent SUI were identified. The success rate at first FU was $89 \%$. At last $\mathrm{FU}$ (median 205 days) the success rate was $83 \%$ $(P<0.01)$. There were no significant differences in success rate between primary and recurrent surgery at first FU. The overall success rate of primary surgery was $86 \%$; for recurrent
\end{abstract}

M. J. van der Doelen $(\varangle) \cdot$ J. P. F. A. Heesakkers

Department of Urology, Radboud University Medical Centre, Geert

Grooteplein Zuid 10 (610), P.O. Box 9101, 6500

HB Nijmegen, The Netherlands

e-mail: Maarten.vanderDoelen@radboudumc.nl

M. I. J. Withagen

Department of Obstetrics and Gynecology, University Medical

Center Utrecht, Utrecht, The Netherlands

M. E. Vierhout

Department of Obstetrics and Gynecology, Radboud University

Medical Centre, Nijmegen, The Netherlands surgery it was $79 \%$. During surgery, 27 bladder injuries occurred (6.2\%), with no significant difference between the two groups. At last FU, 11 patients $(2.6 \%)$ had persistent residual volume, necessitating prolonged clean intermittent selfcatheterization.

Conclusions The results of recurrent surgery to treat recurrent or persistent SUI are still good in experienced hands and do not significantly differ from results of primary surgery. The complication rates are comparable to those of primary surgery.

Keywords Recurrence - Reoperation - Suburethral slings · Urinary incontinence $\cdot$ Stress

$\begin{array}{ll}\text { Abbreviations } \\ \text { AH } & \text { Abdominal hysterectomy } \\ \text { BMI } & \text { Body mass index } \\ \text { CISC } & \text { Clean intermittent self-catheterization } \\ \text { HRT } & \text { Hormone replacement therapy } \\ \text { MUI } & \text { Mixed urinary incontinence } \\ \text { MUS } & \text { Midurethral sling } \\ \text { OAB } & \text { Overactive bladder } \\ \text { POP } & \text { Pelvic organ prolapse } \\ \text { SPC } & \text { Suprapubic catheter } \\ \text { SUI } & \text { Stress urinary incontinence } \\ \text { SVH } & \text { Supravaginal hysterectomy } \\ \text { TOT } & \text { Transobturator tape } \\ \text { TUC } & \text { Transurethral catheter } \\ \text { TUI } & \text { Transurethral injection } \\ \text { TVT } & \text { Tension-free vaginal tape } \\ \text { UI } & \text { Urinary incontinence } \\ \text { UTI } & \text { Urinary tract infection } \\ \text { VH } & \text { Vaginal hysterectomy }\end{array}$




\section{Introduction}

Stress urinary incontinence (SUI) is the complaint of involuntary loss of urine on physical exertion, or on sneezing or coughing [1]. Nowadays, there are plenty of treatments for SUI. The mid-urethral sling (MUS) is currently the gold standard treatment for SUI, with high success rates of approximately $77-85 \%[2,3]$.

Nonetheless, a subgroup of patients experience recurrent or persistent SUI after treatment of their complaints and request retreatment. An estimated $8-17 \%$ of the women who have undergone surgical therapy for urinary incontinence, are likely to undergo a second surgical treatment within $8-10$ years [4, 5].

There are several options for the management of recurrent SUI, including repeating the previously performed procedure, MUS, Burch colposuspension, and bulking agent injections $[6,7]$. Unfortunately, performing a recurrent procedure attempting to solve SUI does not guarantee complete dryness.

To date, there have been limited data on the optimal approach to recurrent SUI, in particular for recurrent SUI after more than two surgical procedures. The aim of this study was to compare cure rates and complication rates in patients who had undergone primary and recurrent surgery for SUI.

\section{Materials and methods}

\section{Patient selection}

We conducted a retrospective cohort study including all medical records of the patients who underwent surgical SUI treatment between January 2002 and March 2010 in the departments of gynecology and urology of the Radboud University Medical Centre, a tertiary referral center in the Netherlands.

Patient enrolment is shown in Fig. 1. A total of 541 patients were identified. All patients had, predominantly, SUI. SUI was defined as the complaint of involuntary loss of urine on physical exertion, or on sneezing or coughing [1]. Exclusion criteria were patients with predominantly overactive bladder (OAB) symptoms $(n=12)$, neurogenic bladder of various causes $(n=29)$, patients with a neobladder $(n=2)$, patients who had last undergone surgery elsewhere $(n=19)$, and patients without postoperative follow-up $(n=3)$. Patients who had undergone a xenograft sling $(n=37)$ were excluded because of the experimental setting of the surgery in a research setting.

Every patient was added to our database only once, even if the patient underwent surgery in our hospital more than once. In recurrent cases, only the result of the last surgical procedure was analyzed.
Study outcomes

The primary outcome of the study was the cure rate based on findings during physical assessment and patient statements at follow-up at six weeks and at the latest available follow-up. Subjective cure/improvement was assessed by an open question during clinical visits and a standardized questionnaire. Objective cure/improvement was tested by a cough test with a full bladder, voiding diary or micturition diary, and a urodynamic investigation if indicated. SUI was considered to have been cured when episodes of involuntary urine leakage during stressful activities according to the patient remained absent and there was no demonstrable SUI during physical examination. Improvement was defined as a significant decrease in urine leakage without further treatment. Failure to cure SUI was classified as unchanged or deteriorated leakage of urine. An unchanged condition was defined as persistent SUI after surgery for SUI. If the urine leakage due to SUI worsened according to the patient, this was classified as deterioration.

Secondary outcomes included the number of peri-operative and postoperative complications, including vaginal tape exposure, pain, and longer term voiding dysfunction.

Surgical procedures, postoperative care, and follow-up

Possible surgical procedures were Burch colposuspension, tension-free vaginal tape (TVT), transobturator tape (TOT), minitape, and periurethral bulking agents.

Operative time and blood loss were measured in every surgical procedure for SUI. Substantial blood loss was defined as more than $500 \mathrm{ml}$.

If another tape had been placed previously, the tape placed during the primary operation was not specifically identified or removed if it was still in place and not hampering the secondary procedure. Intraoperative urethrocystoscopy was performed during all TVT and minitape operations to ensure that no damage had occurred to the urethra or bladder during the procedure.

Among the patients who received a TVT, a TOT or a minitape, a transurethral catheter (TUC) was inserted and kept in position for at least one day.

When performing a Burch colposuspension, a suprapubic catheter (SPC) was inserted in every patient during surgery under direct visualization by intraoperative cystoscopy. Voiding attempts were started one day postoperatively after TVT, TOT, and minitape surgery, and two days postoperatively after Burch colposuspension. If the patient was able to void well and the postvoid residual volume was less than $100 \mathrm{ml}$ on two consecutive occasions, the SPC or TUC was removed and the patient was discharged. In the case of unsatisfactorily voiding, clean intermittent self-catheterization (CISC) was started or the patient was discharged with a TUC or SPC in 
Fig. 1 Patient enrolment

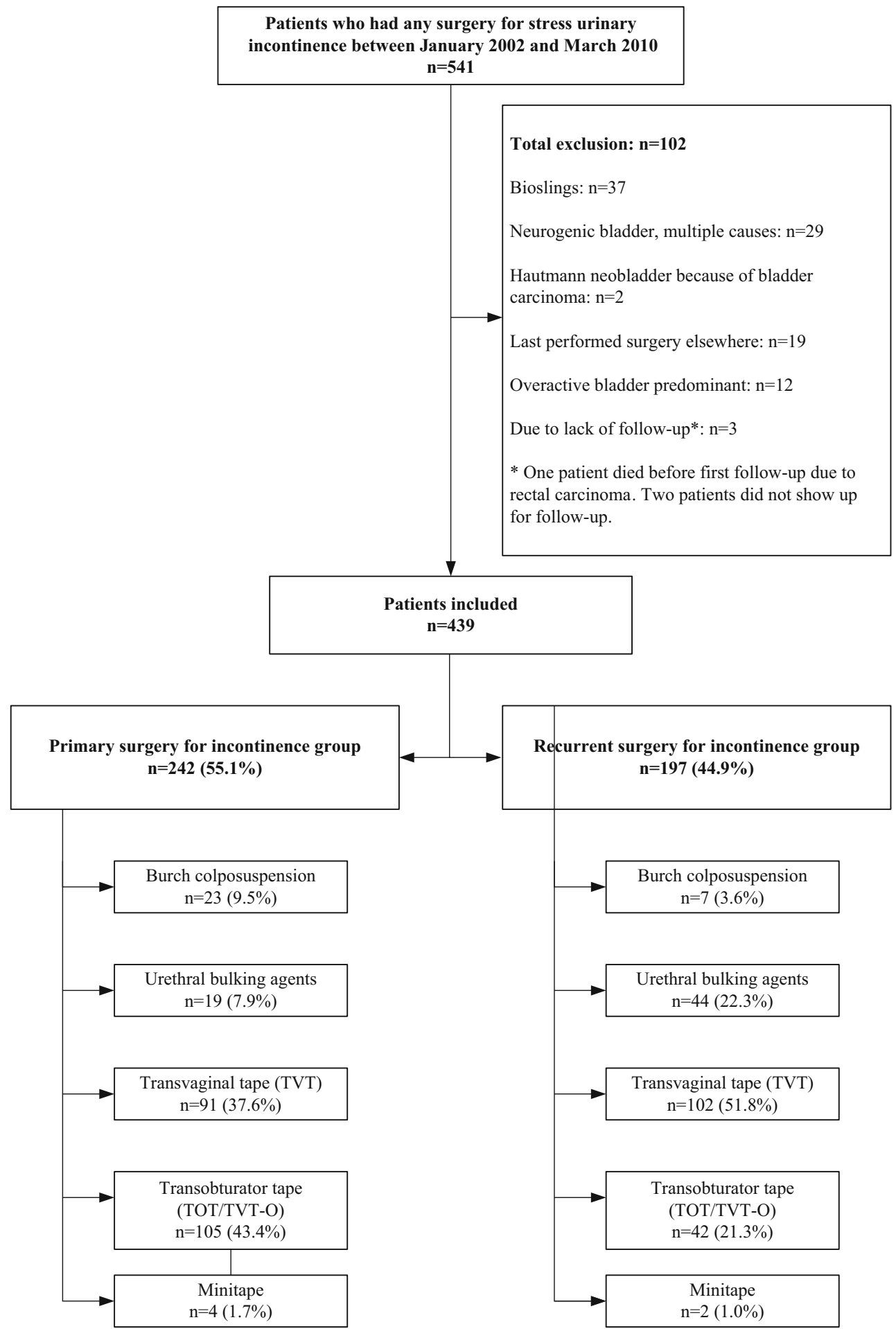

situ and checked after seven days. If the patient was unable to void well at that time, the catheter was kept in place for another week or the patient started CISC and was regularly checked in our hospital.

The periurethral bulking injections were given with local anesthesia and injected into the submucosa through the urethra under direct urethroscopic guidance. Three to four deposits were placed at positions $0.5-1 \mathrm{~cm}$ distal to the bladder neck. Patients were discharged after successful voiding without significant postvoid residual urine (less than $100 \mathrm{ml}$ ).

Patients were assessed at approximately six weeks and three months postoperatively, and thereafter if necessary. Median time between surgery and the last follow-up was 205 days. 
The cure rate was assessed by the observation of involuntary leakage from the urethra synchronous with coughing and also based on patient statements.

\section{Statistical analysis}

In accordance with Dutch law, retrospective observational studies are exempt from submission for approval to a medical ethics review committee. However, the principles of the Declaration of Helsinki were followed. We used the Mann-Whitney $U$ test for independent variables without a normal distribution and the Chi-squared test for categorical variables.

All statistical analyses were performed using SPSS software for Windows (version 17.0; SPSS, Chicago, IL, USA), with $P$ values $<0.05$ considered statistically significant.

\section{Results}

Patient population

A total of 439 patients met the inclusion criteria, of whom 242 had had primary anti-incontinence surgery and 197 recurrent surgery.

Of the 439 patients included, $40 \%(n=176)$ were treated by gynecologists and $60 \%(n=263)$ were treated by urologists. Urologists treated $85 \%(n=167)$ of the recurrent SUI cases.

Baseline characteristics

Patient characteristics are listed in Table 1. Patients with recurrent SUI symptoms had more frequent voiding difficulties

Table 1 Baseline characteristics

\begin{tabular}{|c|c|c|c|c|c|c|c|c|c|c|}
\hline \multirow[b]{3}{*}{ Age at time of surgery, years } & \multicolumn{3}{|c|}{ Complete cohort $(N=439)$} & \multicolumn{3}{|c|}{ Primary surgery group $(n=242)$} & \multicolumn{3}{|c|}{$\begin{array}{l}\text { Recurrent surgery group } \\
(n=197)\end{array}$} & \multirow[t]{2}{*}{$P$} \\
\hline & \multirow{2}{*}{$\begin{array}{l}\text { Number } \\
439\end{array}$} & \multicolumn{2}{|c|}{$\begin{array}{l}\text { Median }[\text { range] or } \\
\text { mean } \pm \mathrm{SD} \text { or number } \\
(\%)\end{array}$} & \multirow{2}{*}{$\begin{array}{l}\text { Number } \\
242\end{array}$} & \multicolumn{2}{|c|}{$\begin{array}{l}\text { Median [range] or } \\
\text { mean } \pm \mathrm{SD} \text { or number } \\
(\%)\end{array}$} & \multirow{2}{*}{$\begin{array}{l}\text { Number } \\
197\end{array}$} & \multicolumn{2}{|c|}{$\begin{array}{l}\text { Median [range] or } \\
\text { mean } \pm \mathrm{SD} \text { or number } \\
(\%)\end{array}$} & \\
\hline & & 59 & {$[22-94]$} & & 56 & {$[38-94]$} & & 62 & {$[22-92]$} & $<0.01^{*}$ \\
\hline Parity, number & 361 & 2 & {$[0-6]$} & 217 & 2 & {$[(0-6]$} & 144 & 2 & {$[0-6]$} & $0.16^{*}$ \\
\hline Menopause and/or using HRT & 388 & 250 & $(64.4)$ & 213 & 115 & $(54.0)$ & 175 & 135 & 77.1 & $<0.01 * *$ \\
\hline BMI, $\mathrm{kg} / \mathrm{m}^{2}$ & 426 & 27.2 & \pm 5.0 & 235 & 26.7 & \pm 4.8 & 191 & 27.9 & \pm 5.1 & $0.03^{*}$ \\
\hline Smoking & 421 & 84 & $(19.1)$ & 231 & 46 & $(19.9)$ & 190 & 38 & $(20.0)$ & $0.98^{* *}$ \\
\hline Current respiratory disease & 439 & 81 & $(18.5)$ & 242 & 41 & $(16.9)$ & 197 & 40 & $(20.3)$ & $0.37 * *$ \\
\hline \multicolumn{11}{|l|}{ Surgical history } \\
\hline \multicolumn{11}{|l|}{ POP surgery } \\
\hline$\geq 1$ surgery for POP & 439 & 94 & $(21.4)$ & 242 & 34 & $(14.0)$ & 197 & 60 & $(30.5)$ & $<0.01 * *$ \\
\hline$\geq 2$ surgeries for $\mathrm{POP}$ & 439 & 25 & $(5.7)$ & 242 & 7 & $(2.9)$ & 197 & 18 & $(9.1)$ & $0.01 * *$ \\
\hline \multicolumn{11}{|l|}{ SUI surgery } \\
\hline$\geq 1$ surgery for $\mathrm{SUI}$ & 439 & 197 & $(44.9)$ & 242 & - & - & 197 & 197 & $(100.0)$ & - \\
\hline$\geq 2$ surgeries for $\mathrm{SUI}$ & 439 & 97 & $(22.1)$ & 242 & - & - & 197 & 97 & $(49.2)$ & - \\
\hline$\geq 3$ surgeries for $\mathrm{SUI}$ & 439 & 38 & (8.7) & 242 & - & - & 197 & 38 & $(19.3)$ & - \\
\hline Hysterectomy & 439 & 140 & $(31.9)$ & 242 & 59 & $(24.4)$ & 197 & 81 & $(41.1)$ & $<0.01 * *$ \\
\hline $\mathrm{AH}$ and $\mathrm{SVH}$ & 433 & 87 & $(20.1)$ & 241 & 32 & $(13.3)$ & 192 & 55 & $(28.6)$ & $<0.01 * *$ \\
\hline VH & 433 & 48 & $(11.1)$ & 241 & 26 & $(10.8)$ & 192 & 22 & $(11.5)$ & $0.83 * *$ \\
\hline MUI & 437 & 190 & $(43.5)$ & 241 & 99 & $(41.1)$ & 196 & 91 & $(46.4)$ & $0.26^{* *}$ \\
\hline \multicolumn{11}{|l|}{ Micturition frequency } \\
\hline Daily frequency & 393 & 8 & {$[3-20]$} & 217 & 8 & {$[4-20]$} & 176 & 8 & {$[3-17]$} & $0.84^{*}$ \\
\hline Nightly frequency & 399 & 1 & {$[0-6]$} & 222 & 1 & {$[0-5]$} & 177 & 2 & {$[0-6]$} & $0.22 *$ \\
\hline Recurrent UTI & 429 & 104 & $(24.2)$ & 235 & 45 & $(19.1)$ & 194 & 59 & $(30.4)$ & $<0.01 * *$ \\
\hline Preoperative use of a CISC or TUC & 439 & 19 & $(4.3)$ & 242 & 3 & $(1.2)$ & 197 & 16 & $(8.1)$ & $<0.01 * *$ \\
\hline
\end{tabular}

$H R T$ hormone replacement therapy, $B M I$ body mass index, $S U I$ stress urinary incontinence, $A H$ abdominal hysterectomy, $S V H$ supravaginal hysterectomy, $V H$ vaginal hysterectomy, MUI mixed urinary incontinence, UTI urinary tract infection, CISC clean intermittent self-catheterization, TUC transurethral catheter

*Calculated using the Mann-Whitney $U$ test

**Calculated using the Chi-squared test 
and used TUC or performed CISC more frequently (1.2\% vs $8.1 \% ; P<0.01)$.

Peri-operative outcomes

Peri-operative data are listed in Table 2.

In $29 \%$ of cases the incontinence surgery was combined with other surgery, such as pelvic organ prolapse (POP) surgery or removal of previous tapes. After the exclusion of concomitant surgeries, we found seven cases $(5.2 \%)$ of excessive bleeding during recurrent procedures carried out to treat SUI and $10(5.7 \%)$ during primary procedures.

One urethral lesion was observed in a patient with an incorrect location of her previous TVT sling, which was removed during the same surgery. The integrity of the urethra was restored and the patient received long-term indwelling catheterization.

All 27 (6.2\%) bladder injuries occurred during TVT surgery; however, there was no significant difference between the two groups. All bladder lesions were recognized intraoperatively by cystoscopy. If a bladder lesion was noticed, the sling was removed, the trocar was replaced and the sling reinserted. There was no prolonged hospital stay in these patients.
Postoperative outcomes

Postoperative data and long-term data are listed in Table 3.

Postoperative complications before discharge were hematoma $(n=4)$, subsequent bleeding $(n=2)$, urinary tract infection during hospital stay $(n=8)$, and pain $(n=22)$. Re-interventions $(n=4)$ were needed in cases of peri-operative bleeding $(n=1)$, postoperative bleeding $(n=2)$, and retensioning of a TVT $(n=$ 1). Postoperative pain was observed significantly more frequently in patients who received concomitant surgery (68\% vs $32 \% ; P<0.01)$ and did not occur significantly more often in patients who underwent recurrent surgery $(27 \%$ vs $73 \% ; P=$ 0.09). Most reported postoperative complaints of pain were located inguinally after TOT $(n=11 ; 7 \%)$ and suprapubically after Burch colposuspension $(n=7 ; 23 \%)$. At first follow-up (six weeks postoperatively), $64 \%(n=14)$ of the 22 patients with postoperative pain did not have pain anymore. At last follow-up, $77 \%(n=17)$ of the 22 patients with postoperative pain no longer had pain.

Before discharge, 53 patients $(12.6 \%)$ were unable to void properly or had post-void residual of more than $100 \mathrm{ml}$. Most patients were able to void well after one week without postvoid residual. Eleven patients $(2.6 \%)$ had persistent

Table 2 Peri-operative data and complications

\begin{tabular}{|c|c|c|c|c|c|c|c|c|c|c|}
\hline \multirow[b]{3}{*}{ Concomitant surgery } & \multicolumn{3}{|c|}{ Complete cohort $(N=439)$} & \multicolumn{3}{|c|}{ Primary surgery group ( $n=242)$} & \multicolumn{3}{|c|}{ Recurrent surgery group $(n=197)$} & \multirow{3}{*}{$\begin{array}{l}P \\
0.39^{*}\end{array}$} \\
\hline & \multirow{2}{*}{$\begin{array}{l}\text { Number } \\
439\end{array}$} & \multicolumn{2}{|c|}{$\begin{array}{l}\text { Median [p5-p95] or } \\
\text { number (\%) }\end{array}$} & \multirow{2}{*}{$\begin{array}{l}\text { Number } \\
242\end{array}$} & \multicolumn{2}{|c|}{$\begin{array}{l}\text { Median }[\mathrm{p} 5-\mathrm{p} 95] \text { or } \\
\text { number }(\%)\end{array}$} & \multirow{2}{*}{$\begin{array}{l}\text { Number } \\
197\end{array}$} & \multicolumn{2}{|c|}{$\begin{array}{l}\text { Median }[\mathrm{p} 5-\mathrm{p} 95] \text { or } \\
\text { number }(\%)\end{array}$} & \\
\hline & & 129 & $(29.4)$ & & 67 & $(27.7)$ & & 62 & $(31.5)$ & \\
\hline Operating time (skin to skin), $\min ^{\mathrm{a}}$ & 286 & 25 & [9-49] & 160 & 25 & [11-47] & 127 & 25 & {$[6-50]$} & $0.58^{* *}$ \\
\hline Burch colposuspension & 5 & 57 & {$[50-65]$} & 4 & 58 & {$[50-65]$} & 1 & 57 & {$[57-57]$} & $1.00^{* *}$ \\
\hline Urethral bulking agents & 43 & 11 & {$[4-27]$} & 9 & 12 & {$[9-20]$} & 34 & 10 & {$[4-30]$} & $0.45^{* *}$ \\
\hline TVT & 130 & 28 & {$[19-50]$} & 68 & 27 & {$[18-46]$} & 62 & 29 & {$[18-53]$} & $0.67^{* *}$ \\
\hline TVT-O/TOT & 103 & 22 & [11-39] & 74 & 22 & [11-39] & 29 & 28 & {$[15-58]$} & $0.03 * *$ \\
\hline Minitape & 5 & 22 & [17-24] & 4 & 23 & [17-24] & 1 & 20 & {$[20-20]$} & $0.47^{* *}$ \\
\hline Blood loss $(\mathrm{ml})^{\mathrm{a}}$ & 265 & 50 & {$[0-200]$} & 145 & 50 & {$[0-200]$} & 120 & 50 & [0-200] & $0.08^{* *}$ \\
\hline Burch colposuspension & 4 & 150 & {$[50-600]$} & 3 & 150 & {$[50-150]$} & 1 & 600 & {$[600-600]$} & $0.16^{* *}$ \\
\hline Urethral bulking agents & 58 & 0 & {$[0-0]$} & 18 & 0 & {$[0-0]$} & 40 & 0 & {$[0-0]$} & $0.50 * *$ \\
\hline (TVT) & 106 & 50 & {$[10-265]$} & 54 & 50 & {$[10-350]$} & 52 & 50 & {$[10-200]$} & $0.04 * *$ \\
\hline TVT-O/TOT & 96 & 50 & [10-215] & 70 & 50 & {$[10-145]$} & 26 & 50 & {$[10-365]$} & $0.67 * *$ \\
\hline Minitape & 1 & 50 & {$[50-50]$} & 0 & - & - & 1 & 50 & {$[50-50]$} & $-* * *$ \\
\hline \multicolumn{11}{|l|}{ Perioperative complications } \\
\hline Bladder lesion & 439 & 27 & $(6.2)$ & 242 & 11 & $(4.5)$ & 197 & 16 & $(8.1)$ & $0.12 *$ \\
\hline Urethral lesion & 439 & 1 & $(0.25)$ & 242 & 0 & $(0.0)$ & 197 & 1 & $(0.5)$ & $-* * *$ \\
\hline Blood loss $>500 \mathrm{ml}^{\mathrm{a}}$ & 310 & 17 & $(5.5)$ & 175 & 10 & $(5.7)$ & 135 & 7 & $(5.2)$ & $0.84 *$ \\
\hline
\end{tabular}

TVT transvaginal tape, TVT-O/TOT transobturator tape

*Calculated using the Chi-squared test

**Calculated with Mann-Whitney $U$ test

***Not enough valid cases to calculate a $P$ value

${ }^{a}$ Without concomitant surgery 
Table 3 Postoperative data and long-term data

\begin{tabular}{|c|c|c|c|c|c|c|c|c|c|c|}
\hline & \multicolumn{3}{|c|}{ Complete cohort $(N=439)$} & \multicolumn{3}{|c|}{ Primary surgery group $(n=242)$} & \multicolumn{3}{|c|}{$\begin{array}{l}\text { Recurrent surgery group } \\
(n=197)\end{array}$} & \multirow[t]{2}{*}{$P$} \\
\hline & Number & \multicolumn{2}{|c|}{$\begin{array}{l}\text { Median [range] or } \\
\text { number }(\%)\end{array}$} & Number & \multicolumn{2}{|c|}{$\begin{array}{l}\text { Median [range] or } \\
\text { number }(\%)\end{array}$} & Number & \multicolumn{2}{|c|}{$\begin{array}{l}\text { Median [range] or } \\
\text { number }(\%)\end{array}$} & \\
\hline \multicolumn{11}{|l|}{ Postoperative data } \\
\hline Pain $^{\mathrm{a}}$ & 439 & 22 & $(5.0)$ & 22 & 16 & $(72.7)$ & 22 & 6 & $(27.3)$ & $0.09 *$ \\
\hline Pain ${ }^{b}$ & 310 & 7 & $(2.3)$ & 175 & 3 & $(1.7)$ & 135 & 4 & $(3.0)$ & $0.47 * *$ \\
\hline After Burch colposuspension & 5 & 2 & $(40.0)$ & 4 & 2 & $(50.0)$ & 1 & 0 & $(0.0)$ & $1.00 * *$ \\
\hline After TVT & 137 & 2 & $(1.5)$ & 74 & 0 & $(0.0)$ & 63 & 2 & $(3.2)$ & $0.21 * *$ \\
\hline After TOT & 103 & 3 & (2.9) & 74 & 1 & (1.4) & 29 & 2 & $(6.9)$ & $0.19^{* *}$ \\
\hline Hematoma $^{\text {a }}$ & 439 & 4 & $(0.9)$ & 242 & 3 & $(1.2)$ & 197 & 1 & $(0.5)$ & $0.63 * *$ \\
\hline Re-intervention due to complication ${ }^{a}$ & 439 & 4 & $(0.9)$ & 242 & 3 & $(1.2)$ & 197 & 1 & $(0.5)$ & $0.63 * *$ \\
\hline Urinary retention $^{c}$ & 420 & 53 & $(12.6)$ & 239 & 31 & $(13.0)$ & 181 & 22 & $(12.2)$ & $0.80 *$ \\
\hline Temporary & 420 & 42 & $(10.0)$ & 239 & 25 & $(10.5)$ & 181 & 17 & $(9.4)$ & $0.77 *$ \\
\hline Persistent & 420 & 11 & $(2.6)$ & 239 & 6 & $(2.5)$ & 181 & 5 & $(2.8)$ & $0.77^{*}$ \\
\hline Duration of urinary catheter (days) ${ }^{a}$ & 420 & 1 & {$[0-34]$} & 236 & 1 & {$[0-9]$} & 184 & 1 & {$[0-34]$} & $0.10^{* * *}$ \\
\hline Hospital stay (days) ${ }^{\mathrm{a}}$ & 439 & 1 & {$[0-15]$} & 242 & 1 & {$[0-10]$} & 197 & 1 & {$[0-15]$} & $<0.01 * * *$ \\
\hline Hospital stay (days) ${ }^{\text {b }}$ & 310 & 1 & {$[0-15]$} & 175 & 1 & {$[0-8]$} & 135 & 1 & {$[0-15]$} & $0.14^{* * *}$ \\
\hline \multicolumn{11}{|l|}{ Long-term data } \\
\hline \multicolumn{11}{|l|}{ Micturition frequency $^{\mathrm{a}}$} \\
\hline Daily frequency & 191 & 8 & {$[2-20]$} & 105 & 8 & {$[2-20]$} & 86 & 8 & {$[3-20]$} & $0.31 * * *$ \\
\hline Nightly frequency & 187 & 2 & {$[0-7]$} & 103 & 1 & {$[0-7]$} & 84 & 2 & {$[0-5]$} & $0.43 * * *$ \\
\hline De novo OAB symptoms ${ }^{\text {a }}$ & 243 & 52 & $(21.4)$ & 139 & 24 & $(17.3)$ & 104 & 28 & $(26.9)$ & $0.07^{*}$ \\
\hline Pain $^{\mathrm{a}}$ & 431 & 57 & $(13.2)$ & 236 & 38 & $(16.1)$ & 195 & 19 & $(9.7)$ & $0.05^{*}$ \\
\hline Pain ${ }^{b}$ & 308 & 32 & $(10.3)$ & 174 & 21 & (12.1) & 134 & 11 & $(8.1)$ & $0.27^{*}$ \\
\hline After TVT & 136 & 16 & $(11.8)$ & 74 & 9 & $(12.2)$ & 62 & 7 & $(11.3)$ & $0.87^{*}$ \\
\hline After TOT & 102 & 11 & $(10.8)$ & 73 & 8 & $(11.0)$ & 29 & 3 & $(10.3)$ & $0.93 *$ \\
\hline Vaginal exposure $^{a}$ & 439 & 13 & $(3.0)$ & 242 & 8 & $(3.3)$ & 197 & 5 & $(2.5)$ & $0.64 *$ \\
\hline After MUS & 346 & 8 & $(2.3)$ & 200 & 5 & $(2.5)$ & 146 & 3 & $(2.1)$ & $0.79 * *$ \\
\hline After bulking agents & 63 & 5 & (7.9) & 19 & 3 & $(15.8)$ & 44 & 2 & $(4.5)$ & $0.13 * *$ \\
\hline \multicolumn{11}{|l|}{ *Calculated using the Chi-squared test } \\
\hline \multicolumn{11}{|c|}{${ }^{* *}$ Calculated using Fisher's exact test } \\
\hline \multicolumn{11}{|c|}{ ***Calculated using the Mann-Whitney $U$ test } \\
\hline \multicolumn{11}{|c|}{${ }^{a}$ Concomitant surgery included } \\
\hline${ }^{\mathrm{b}}$ Concomitant surgery excluded & & & & & & & & & & \\
\hline
\end{tabular}

retention, necessitating prolonged CISC or an indwelling catheter. Two women wanted lysis of the tape, which was performed. Also, three patients had an extended postoperative transurethral catheter for special reasons (one urethral lesion and two patients with voiding dysfunction after a bladder lesion), but could void normally afterwards.

Pre-operatively, 19 patients had a TUC or a SPC or performed CISC. Directly after surgery four of these patients still had urinary retention and needed to perform CISC. The other 15 patients were able to void spontaneously without considerable residual volume, but ten of them needed a catheter at last follow-up (205 days).

Long-term outcomes

The success rates of primary and recurrent surgery are compared in Table 4.

Overall, $89 \%$ of the patients had relief, either complete or improved, of SUI symptoms at first follow-up. At last followup the success rate was $83 \%$. There were no significant 
Table 4 Success of primary surgery vs recurrent surgery for stress urinary incontinence

\begin{tabular}{|c|c|c|c|c|c|c|c|}
\hline & \multirow[b]{3}{*}{ Number } & \multicolumn{2}{|c|}{$\begin{array}{l}\text { Success at first follow-up } \\
\text { (6 weeks) }\end{array}$} & \multirow{3}{*}{$\begin{array}{l}P \text { value primary surgery } \\
\text { compared with recurrent } \\
\text { surgery* }\end{array}$} & \multicolumn{2}{|c|}{ Success at last follow-up } & \multirow{3}{*}{$\begin{array}{l}P \text { value primary surgery } \\
\text { compared with recurren } \\
\text { surgery* }\end{array}$} \\
\hline & & \multirow{2}{*}{$\begin{array}{l}\text { Number } \\
(\%) \\
\text { Primary } \\
\text { surgery } \\
n=242\end{array}$} & \multirow{2}{*}{$\begin{array}{l}\text { Number (\%) } \\
\text { Recurrent } \\
\text { surgery } \\
n=197\end{array}$} & & \multirow{2}{*}{$\begin{array}{l}\text { Number } \\
(\%) \\
\text { Primary } \\
\text { surgery } \\
n=242\end{array}$} & \multirow{2}{*}{$\begin{array}{l}\text { Number (\%) } \\
\text { Recurrent } \\
\text { surgery } \\
n=197\end{array}$} & \\
\hline & & & & & & & \\
\hline $\begin{array}{l}\text { Burch } \\
\quad \text { colposuspension }\end{array}$ & 30 & $21(91.3)$ & $4(57.1)$ & 0.07 & $20(87.0)$ & $4(57.1)$ & 0.12 \\
\hline $\begin{array}{l}\text { Urethral bulking } \\
\text { agents }\end{array}$ & 63 & $12(63.2)$ & $36(81.8)$ & 0.11 & $9(47.4)$ & $30(68.2)$ & 0.12 \\
\hline TVT & 193 & $84(92.3)$ & $94(92.2)$ & 0.97 & $82(90.1)$ & $88(86.3)$ & 0.41 \\
\hline TVT-O/TOT & 147 & $99(94.3)$ & $34(81.0)$ & 0.01 & $94(89.5)$ & $33(78.6)$ & 0.08 \\
\hline Minitape & 6 & $4(100.0)$ & $2(100.0)$ & 1.00 & $4(100.0)$ & $1(50.0)$ & 0.33 \\
\hline Total & 439 & $220(90.9)$ & $170(86.3)$ & 0.127 & $209(86.4)$ & $156(79.2)$ & 0.046 \\
\hline
\end{tabular}

*Calculated using the Chi-squared test

differences between primary and recurrent surgery at first-follow-up, but at last follow-up the success rate of recurrent surgery was lower (79 \%) than the success rate of $86 \%$ for primary surgery at last follow-up $(P=0.05)$.

Of the 100 patients in whom primary surgery failed, 68 patients had undergone primary surgery with a MUS previously. Most of them were treated with a new MUS (79 \%, $n=$ $54)$; the success rate was $85 \%$. Another $16 \%(n=11)$ were treated using periurethral bulking agents; the success rate was $73 \%$.

Twenty-three patients had undergone primary surgery using periurethral bulking agents previously. Most of them were treated with an MUS ( $78 \%, n=18)$; the success rate was $89 \%$. Another $17 \%(n=4)$ were treated using periurethral bulking agents; the success rate was $75 \%$.

At first follow-up, vaginal exposure was reported in five patients $(2.1 \%)$ after primary surgery and in three patients $(1.5 \%)$ after recurrent surgery $(P=0.74)$.

Urethral mucosal exposure occurred twice after bulking agents and six times after MUS.

At last follow-up, two of these patients had persistent exposure in the vagina and needed re-excision of their MUS ( $n=$ $1)$ and bulking agents $(n=1)$. Five other patients reported new exposure at the last follow-up (three times after bulking agents and twice after MUS), all patients underwent surgical excision.

\section{Discussion}

This manuscript clearly illustrates that the success rates of primary and secondary procedures are acceptable, with no significantly higher rates of complications. In this study we found success rates of $86 \%$ for primary cases and $79 \%$ for recurrent cases. Overall, only $17 \%$ of patients experienced no relief of SUI symptoms six months after surgery.

In a recent review, Pradhan et al. noted a subjective cure rate following MUS for recurrent SUI after any previous surgery of $79 \%$ at follow-up of $30 \pm 29$ months [8]. This cure rate is slightly lower than our success rate of $84 \%$ for surgery with MUS at last follow-up, but other criteria for success were applied in the various studies and the follow-up of our study is much shorter.

We observed a lower cure rate with TOT (79\%) than with the retropubic tape $(86 \%)$ for recurrent SUI. This is in line with the results described in the review by Pradhan et al., although they reported lower success rates $(54 \%)$ than in this study [8]. This could also have been caused by the relatively low number of our patients who underwent TOT to treat recurrent SUI. These results contradict the results of the systematic review by Agur et al., who described TOT as having similar patient-reported and objective cure/improvement rates compared with retropubic TVT in the surgical treatment of women with recurrent SUI [9].

In our study, treatment with bulking agents resulted more often in improvement than in complete dryness (54\% and $8 \%$ respectively). In patients with recurrent SUI symptoms the results with bulking agents were slightly higher (59\% and $9 \%$ respectively). These low percentages are consistent with those of a previous study, in which bulking agents after previous MUS showed a cure rate of $35 \%$ for a median follow-up of 10 months [10]. However, patient satisfaction was relatively high $(77 \%)$ in this study after this minimally invasive procedure. This implies that bulking agents could play a role 
in secondary treatment to improve SUI symptoms after a previous failed procedure, especially when more invasive procedures are not an option.

As we performed only seven Burch colposuspensions in patients with recurrent SUI symptoms, it is difficult to generate proper conclusions.

Stav et al. have argued that de novo OAB symptoms are more common in patients who underwent a repeat MUS to treat persistent or recurrent SUI [2]. In our study, de novo OAB symptoms were seen in $52(21 \%)$ patients, with no significant difference between the two groups.

According to a systematic review, the incidence of de novo urgency after MUS to treat recurrent SUI is between 5.5 and $20 \%$ [8]. A possible explanation for the higher incidence of $27 \%$ (after any recurrent procedure) in our series is the high number of patients who had undergone more than two previous operations to treat SUI. According to Pradhan et al., the rate of urinary tract injuries is $8 \%$ with retropubic sling procedures during repeat continence surgery, while it is $4 \%$ during primary retropubic sling procedures [8]. These results are consistent with those we found in our study ( $8 \%$ and $5 \%$ respectively).

As described above, we found seven cases of excessive bleeding $(2 \%)$ during recurrent procedures carried out to treat SUI. According to the literature, the incidence of excessive bleeding during repeat SUI surgery is $2-7 \%$ [8].

Postoperatively, $12 \%$ of patients had symptoms of voiding dysfunction after recurrent surgery. At last follow-up, 11 patients $(3 \%)$ had persistent residual volumes, necessitating prolonged CISC. There was no significant difference between the patients who underwent primary surgery and the patients who underwent recurrent surgery. Pradhan et al. described the frequency of voiding dysfunction after recurrent surgeries as varying between 4 and $16 \%$ [8].

In our study, success rates of urethral bulking agents and TVT turned out to have significantly worsened by time of the last follow-up visit compared with the first follow-up. Repeat continence surgery is often difficult as a result of retropubic scarring and adhesions with the distortion in anatomy from previous surgeries, which may increase the risks of intraoperative complications [11]. Amaye-Obu and Drutz found that the first surgical approach for the management of SUI is the best, because cure rates decline proportionately with the number of subsequent operations performed [12]. We also found this trend in our data.

The impact of this study is limited by its retrospective design and descriptive nature. It is, therefore, susceptible to recall and interpretation bias. Moreover, since data were collected retrospectively, $30 \%$ of the patients had a follow-up of less than 3 months. Therefore, the postoperative follow-up may have been too short to find recurrences of SUI in some cases.

The fact that our academic hospital is a tertiary care center limits the generalizability of our conclusions. Some patients needed multiple procedures to achieve optimal relief of their SUI symptoms. In our study, nearly $9 \%$ of the patients needed three or more procedures, with a maximum of as many as eight previous procedures $(n=1)$.

Despite these limitations, this study is strengthened by the large number of patients, by the fact that all operations were performed in one center by experienced surgeons using the same surgical techniques, and by the detailed data available from operative and medical records.

\section{Conclusion}

Results of recurrent surgery to treat recurrent or persistent SUI are still good in experienced hands and complication rates are comparable to those of primary surgery. The treatment of choice should always be adapted to the specific patient needs, especially in recurrent cases. In our opinion recurrent SUI symptoms should only be treated with utmost skill and only by trained and experienced surgeons, preferably in a tertiary referral center. Patients should be informed about the potential risks of recurrent SUI surgery, in particular about exposure and voiding difficulties. Our results support the claims of the contemporary literature that retropubic midurethral slings are the first choice in the treatment of SUI symptoms after failure of a previous anti-incontinence operation. Long-term followup is important because of a time-dependent decline in cure rate.

Financia disclaimer/conflict of interest M.J. van der Doelen: none; M.I.J. Withagen: received honoraria for speaking at symposia about the use of mesh from Ethicon; M.E. Vierhout: member of the European Advisory Board on OAB of Astellas; J.P.F.A. Heesakkers: none.

Open Access This article is distributed under the terms of the Creative Commons Attribution License which permits any use, distribution, and reproduction in any medium, provided the original author(s) and the source are credited.

\section{References}

1. Haylen BT, de Ridder D, Freeman RM, Swift SE, Berghmans B, Lee J, Monga A, Petri E, Rizk DE, Sand PK, Schaer GN (2010) An International Urogynecological Association (IUGA)/International Continence Society (ICS) joint report on the terminology for female pelvic floor dysfunction. Int Urogynecol J 21(1):5-26. doi:10.1007/ s00192-009-0976-9

2. Stav K, Dwyer PL, Rosamilia A, Schierlitz L, Lim YN, Lee J (2010) Risk factors of treatment failure of midurethral sling procedures for women with urinary stress incontinence. Int Urogynecol J 21(2): 149 155. doi:10.1007/s00192-009-1020-9

3. Nilsson CG, Palva K, Aarnio R, Morcos E, Falconer C (2013) Seventeen years' follow-up of the tension-free vaginal tape procedure for female stress urinary incontinence. Int Urogynecol J 24(8): 1265-1269. doi:10.1007/s00192-013-2090-2 
4. Fialkow M, Symons RG, Flum D (2008) Reoperation for urinary incontinence. Am J Obstet Gynecol 199(5):546.e1-546.e8. doi:10. 1016/j.ajog.2008.04.047

5. Denman MA, Gregory WT, Boyles SH, Smith V, Edwards SR, Clark AL (2008) Reoperation 10 years after surgically managed pelvic organ prolapse and urinary incontinence. Am J Obstet Gynecol 198(5):555.e1-555.e5. doi:10.1016/j.ajog.2008.01.051

6. Scarpero HM, Dmochowski RR (2004) Sling failures: what's next? Curr Urol Rep 5(5):389-396

7. De Ridder D (2009) Editorial comment on: tension-free vaginal tape in the management of recurrent urodynamic stress incontinence after previous failed midurethral tape. Eur Urol 55(6):1455-1456. doi:10. 1016/j.eururo.2009.03.026

8. Pradhan A, Jain P, Latthe PM (2012) Effectiveness of midurethral slings in recurrent stress urinary incontinence: a systematic review and meta-analysis. Int Urogynecol J 23(7):831-841. doi:10.1007/ s00192-012-1803-2
9. Agur W, Riad M, Secco S, Litman H, Madhuvrata P, Novara G, Abdel-Fattah M (2013) Surgical treatment of recurrent stress urinary incontinence in women: a systematic review and meta-analysis of randomised controlled trials. Eur Urol 64(2):323-336. doi:10.1016/ j.eururo.2013.04.034

10. Lee HN, Lee YS, Han JY, Jeong JY, Choo MS, Lee KS (2010) Transurethral injection of bulking agent for treatment of failed midurethral sling procedures. Int Urogynecol J 21(12):1479-1483. doi: 10.1007/s00192-010-1224-Z

11. Rezapour M, Ulmsten U (2001) Tension-Free vaginal tape (TVT) in women with recurrent stress urinary incontinence-a long-term follow up. Int Urogynecol J Pelvic Floor Dysfunct 12 [Suppl 2]:S9-S11

12. Amaye-Obu FA, Drutz HP (1999) Surgical management of recurrent stress urinary incontinence: a 12-year experience. Am J Obstet Gynecol 181(6):1296-1307, discussion 1307-1309 